\title{
HEALTH-RELATED QUALITY OF LIFE IN PATIENTS WITH HAEMOPHILIA AND ITS ASSOCIATION WITH DEPRESSIVE SYMPTOMS: A STUDY IN CROATIA AND SLOVENIA
}

\author{
Martina Bago" ${ }^{1}$, Ana Butkovic ${ }^{2}$, Barbara Faganel Kotnik ${ }^{3,4}$, Ivana Prga ${ }^{1}$, Vesna Bačić Vrca ${ }^{5,6}$, \\ Silva Zupančić Šalek ${ }^{7,8}$ \& Irena Preloznik Zupan ${ }^{4,9}$ \\ ${ }^{I}$ Reference Centre for Pharmacoepidemiology, Andrija Stampar Teaching Institute of Public Health, Zagreb, Croatia \\ ${ }^{2}$ Faculty of Humanities and Social Sciences, University of Zagreb, Zagreb, Croatia \\ ${ }^{3}$ Division of Paediatrics, University Medical Centre Ljubljana, Ljubljana, Slovenia \\ ${ }^{4}$ Faculty of Medicine, University of Ljubljana, Ljubljana, Slovenia \\ ${ }^{5}$ Hospital Pharmacy, Clinical Hospital Dubrava, Zagreb, Croatia \\ ${ }^{6}$ Faculty of Pharmacy and Biochemistry, University of Zagreb, Zagreb, Croatia \\ ${ }^{7}$ Department of Internal Medicine, University Hospital Centre Zagreb, Zagreb, Croatia \\ ${ }^{8}$ Faculty of Medicine, University of Zagreb, Zagreb, Croatia \\ ${ }^{9}$ Division of Internal Medicine, University Medical Center Ljubljana, Ljubljana, Slovenia
}

received: 28.4.2021; $\quad$ revised: 28.6.2021; $\quad$ accepted: 5.7.2021

\section{SUMMARY}

Background: There are only a few studies in patients with haemophilia (PWH) that examined both quality of life and depressive symptoms, with only few studies examining their association. Aim of this study was to examine the association between depressive symptoms and health-related quality of life (HRQoL) in PWH from Croatia and Slovenia.

Subjects and methods: A total of 112 adult PWH on prophylactic (73\%) or on-demand (27\%) treatment were included in the study (median age 46 years, range 18-73 years). Depressive symptoms were assessed with BDI-II, HRQoL with SF-36v2, demographic and socioeconomic data were collected using a questionnaire, and clinical data were obtained from medical records.

Results: All HRQoL scores were significantly negatively correlated with BDI-II in the -0.42 to -0.70 range (all $p<0.05$ ). Sociodemographic and clinical variables explained 28-51\% of HRQoL variance scores. Depressive symptoms explained additional variance for six HRQoL domain scores, with incremental variance being larger for mental domain scores (ranging between 10$27 \%$ ), and for Mental Component Summary score (26\%).

Conclusions: This study's findings support that having depressive symptoms is associated with HRQoL of PWH, more so in the mental health than in the physical health domains.

Key words: depression - haemophilia A - haemophilia B - quality of life

\section{INTRODUCTION}

Haemophilia is a rare chronic disease caused by coagulation factor VIII (haemophilia A) or IX (haemophilia B) deficiency. Patients with haemophilia (PWH) are facing many physical problems which influence both their physical and psychological health. The evaluation of health related quality of life (HRQoL) is essential for a full assessment of the influence an illness, such as haemophilia, has on patients' lives (Limperg et al. 2017). HRQoL is therefore increasingly recognized as a significant component of modern haemophilia management and as an important health outcome measure in haemophilia, which may help in improving treatment strategies (Varaklioti et al. 2014).

Several studies investigated HRQoL in PWH and these studies have consistently shown that PWH have lower HRQoL than the general population (Castaño et al. 2017, Cavazza et al. 2016, Kempton et al. 2018a, Naous et al. 2019, Miners et al. 1999, Poon et al. 2012) and that patients with severe haemophilia have lower
HRQoL than patients with moderate or mild haemophilia (Miners et al. 1999, Poon et al. 2012). Since HRQOL is an individual's perceived physical and mental health over time (Centers for Disease Control and Prevention 2000), it depends on the individual's perception and mental state. It has been shown that psychological variables (anxiety, anger, optimism and depression) are the strongest determinants of well-being (satisfaction, health and somatic complaints) in $\mathrm{PWH}$ (Triemstra et al. 1998).

Symptoms of anxiety and depression in patients with chronic diseases have an impact on the control of a chronic disease, quality of life and general health (Gerontoukou et al. 2015). Depressive symptoms have been found to differentiate between groups even among patients with chronic symptoms such as watery eye symptoms (Mandić et al. 2018). Between 9.3\% and $23 \%$ of patients with one or more chronic physical disease have comorbid depression, and the likelihood of having depression is higher in the presence of a chronic physical disease (Moussavi et al. 2007). 
Similar numbers have been found for PWH with prevalence of depression ranging from $8 \%$ to $37 \%$ (Kempton et al. 2018a, Miners et al. 1999, Canclini et al. 2003, Bago et al. 2020, Pinto et al. 2018b, Tran et al. 2017, Barlow et al. 2007, Iannone et al. 2012). Studies in PWH showed that depressive symptoms are associated with more urgent hospital visits due to haemophilia (Pinto et al. 2018a), more bleeding episodes (Iannone et al. 2012, Pinto et al. 2018a), more affected joints (Pinto et al. 2018a), hepatitis C (Barlow et al. 2007), greater pain (Kempton et al. 2018a), low self-esteem (Canclini et al. 2003) and worse quality of life (Pinto et al. 2018a).

There are only a few studies in PWH that examined both quality of life (QoL) and depressive symptoms. In addition, in some of those few studies which included measures of QoL and depressive symptoms, their association was not examined. Examples are the Pain, Functional Impairment, and Quality of Life (P-FiQ) study on 381 PWH which included SF-36 as a measure of HRQoL and EQ-5D-5L as a measure of anxiety/depression (Kempton et al. 2018a, Buckner et al. 2018, Batt et al. 2018), and Castaño et al. study on 59 PWH which included SF-36 as a measure of HRQoL and PHQ-9 as a measure of depressive symptoms (Castaño et al. 2017). In those studies, the association between depressive symptoms and HRQoL was not examined.

On the other hand, Kim et al. investigated the impact of depression and personality on QoL in 53 Korean patients with severe haemophilia (Kim et al. 2013). They assessed depression with Beck Depression Inventory (BDI) and QoL with the World Health Organization Quality of Life - abbreviated version which gives scores on four domains: physical health, psychological, social relationships and environment. BDI scores were significantly and negatively associated with all four domains of the QoL in both correlational and regression analyses. Rambod et al. collected data on $103 \mathrm{PWH}$ in Iran with the haemophilia-specific QoL measure (Haem-A-QoL) and Depression Anxiety Stress Scales (DASS) (Rambod et al. 2018). In multiple regression analysis hepatitis status, severity of disease, stress, depression, anxiety, severity of pain, and having bleeding were included as predictors and $47 \%$ of HRQoL variance was explained by severity of pain and depression.

The aim of this study was to examine the association between depressive symptoms measured with Beck Depression Inventory (BDI-II) and HRQoL measured with The SF-36v2 ${ }^{\circledR}$ Health Survey (SF-36v2) in PWH from Croatia and Slovenia. Although SF-36 is the most common HRQoL measure, its association with depressive symptoms in PWH has not been examined so far. PWH are an especially vulnerable population in terms of depression and low HRQoL because they have to deal with bleedings, mostly in joints, which can affect their mobility and cause disability. Therefore, examining the association between depressive symptoms and HRQoL in PWH is important both as a research question, but also in order to better understand what PWH are dealing with and to better tailor their treatment. Based on previous findings (Kim et al. 2013, Rambod et al. 2018), we hypothesized that depressive symptoms will negatively and significantly predict HRQoL variance in PWH.

\section{SUBJECT AND METHODS}

\section{Data collection}

Data for this cross-sectional study were collected in the National Haemophilia Centre at University Hospital Centre Zagreb, Croatia and in the National Haemophilia Centre at University Medical Centre Ljubljana, Slovenia from April 2018 to October 2019. The study conduction was approved by the Ethics Committee of the University Hospital Centre Zagreb and National Medical Ethics Committee of the Republic of Slovenia. The study was conducted in line with the Declaration of Helsinki. Patients who agreed to participate in the study were asked to sign informed consent before filling in the questionnaire.

\section{Study sample}

Eligibility criteria were: male patients with haemophilia A or B aged 18 or older who were currently using prophylactic or on-demand treatment for at least one year before the recruitment. Non-inclusion criteria were the presence of inhibitors and cognitive impairment because they could have influenced the study results. There were 112 participants in the final sample (see Table 1). Median age of participants was 46 years (range 18-73). There were 65 (58\%) participants from Croatia and 47 (42\%) participants from Slovenia. From the 112 participants in the study, $82(73 \%)$ were receiving prophylactic and $30(27 \%)$ on-demand treatment. Most of the participants had haemophilia A (85\%) and severe type of haemophilia (78\%). The majority of participants finished secondary education $(56 \%)$, lived with other people in the household $(81 \%)$ and were within the working age range (67\%). Participants in this study mainly had chronic diseases beside haemophilia $(62 \%)$ with $71 \%$ having arthropathy, $12 \%$ arthritis, and $10 \%$ hepatitis C $(37 \%$ had hepatitis $\mathrm{C}$ in the past but were cured). Since most of the participants were on prophylaxis, only $30 \%$ had 12 or more bleeding episodes in the previous year and only $16 \%$ were hospitalized in the last six months.

\section{Sample size}

Sample size was determined for multivariate regression analysis with power value set at 0.80 and alpha level value se at $p<0.05$. Using PASS 15 Software (PASS 15 2017) a 77-participants sample size was determined. 
Table 1. Sample characteristics

\begin{tabular}{lc}
\hline Characteristic & $\mathrm{n}(\%)$ \\
\hline Treatment & \\
Prophylaxis & $82(73 \%)$ \\
On-demand & $30(27 \%)$ \\
Education & \\
Primary & $17(15 \%)$ \\
Secondary & $63(56 \%)$ \\
Tertiary & $32(29 \%)$ \\
Household & \\
Alone & $21(19 \%)$ \\
With someone & $91(81 \%)$ \\
Work status & \\
Working age & $75(67 \%)$ \\
Retired & $37(33 \%)$ \\
Diagnosis & \\
Haemophilia A & $95(85 \%)$ \\
Haemophilia B & $16(15 \%)$ \\
Severity & \\
Mild & $11(10 \%)$ \\
Moderate & $13(12 \%)$ \\
Severe & $86(78 \%)$ \\
Bleeding episodes & \\
11 or less & $77(71 \%)$ \\
12 or more & \\
Hospitalizations & \\
No & $35(70 \%)$ \\
Yes & $34(30 \%)$ \\
Chronic diseases & \\
No & $94(84 \%)$ \\
Yes & $18(16 \%)$ \\
Arthritis diagnosis & \\
No & \\
Yes & \\
Hepatitis C diagnosis & \\
No & \\
Cured & \\
Yes & \\
\hline
\end{tabular}

\section{Instruments}

Demographic and socioeconomic data were collected using a self-reported questionnaire designed for this study. The questionnaire provided information about age, educational level (finished primary, secondary or tertiary education), household status $(0=$ living alone, $1=$ living with other people), work status $(0=$ working age, 1 = retired) and monthly household income (nine categories with category 5 representing average income).

Clinical data were obtained from the medical records and provided information about the diagnosis ( 1 = haemophilia A, 2 = haemophilia $\mathrm{B})$, severity $(0=$ mild, $1=$ moderate, $2=$ severe $)$, bleeding episodes during the previous 12 months $(1=11$ or less, $2=12$ or more), hospitalizations in the previous 6 months $(0=$ no, $1=$ yes $)$, other chronic diseases $(0=$ none, $1=1$ or more), arthritis diagnosis $(0=$ no, $1=$ yes $)$, hepatitis $C$ diagnosis ( $0=$ no, $1=$ cured, $2=$ yes $)$ and arthropathy diagnosis $(0=$ no, $1=$ yes $)$. Information about some of the clinical data was not available for all participants: diagnosis was missing for one participant, severity of haemophilia for two participants, other chronic conditions for six participants, arthritis diagnosis for five participants, hepatitis $\mathrm{C}$ diagnosis for eight participants and arthropathy diagnosis for three participants.

Depressive symptoms were assessed with BDI-II, an instrument for indicating the presence and degree of depressive symptoms consistent with the Diagnostic and Statistical Manual of Mental Disorders, Fourth Edition (DSM-IV) (Beck et al. 1996). BDI-II is a self-reported instrument with 21 items evaluating 21 symptoms of depression and each item is rated on a four-point scale. Total score ranges from 0 to 63 and higher score indicates higher severity of depressive symptoms. Naklada Slap provided BDI-II in Croatia and NCS Pearson Inc. in Slovenia. Cronbach alpha reliability in this study was 0.90 in the total sample, 0.91 in Croatian sample and 0.85 in Slovenian sample.

HRQoL was assessed with SF-36v2, a generic measure of HRQoL (Maruish 2011). SF-36v2 is a short-form self-administered health survey with 36 items corresponding to eight dimensions of HRQoL: physical functioning (PF), role-physical (RP), bodily pain (BP), general health $(\mathrm{GH})$, vitality $(\mathrm{VT})$, social functioning (SF), role-emotional (RE), and mental health (MH). The Physical Component Summary (PCS) score and the Mental Component Summary (MCS) score are obtained using those eight dimensions. OptumInsight Life Sciences, Inc. provided the use of SF-36v2 in Croatian and Slovenian as well as the scoring software. Cronbach alpha reliabilities of the domain raw scores ranged between 0.75 and 0.95 in the total sample, between 0.76 and 0.95 in Croatian sample and between 0.66 and 0.96 in Slovenian sample.

\section{Statistical analysis}

All statistical analyses were run in SPSS 26.0 (IBM Corp. 2019). We first examined descriptive statistics, and normality of distributions. Although KolmogorovSmirnov tests indicated that HRQoL distributions were different from the normal distribution, the values of skewness and kurtosis coefficients were in the range of the suggested values for the distribution to be considered approximately normal (Ryu 2011). Next, we ran correlational analysis using Pearson correlation coefficients for two continuous variables and point-biserial correlation coefficients for dichotomous and continuous variables, and regression analyses. Statistical significance was set at $\mathrm{p}<0.05$. 


\section{RESULTS}

Descriptive statistics for HRQoL and correlations with depressive symptoms, socio-demographic and clinical variables are presented in Table 2. Depressive symptoms were not normally distributed in the sample, participants' scores were in the $0-29$ range with $\mathrm{Mo}=1$. Participants in this study had better scores in the mental health (VT, SF, RE, MH) than in the physical health (PF, RP, BP, $\mathrm{GH}$ ) domains, and higher MCS than PCS score. Almost all HRQoL norm-based scores of our participants were below 50, indicating HRQoL below the US average.

All HRQoL scores were significantly negatively correlated with BDI-II in the -0.42 to -0.70 range (all $\mathrm{p}<0.05)$. Point-biserial correlation was calculated for household and work and all clinical variables. The socio-demographic and clinical variables positively correlated with HRQoL were income, education, household and arthritis. The negatively correlated variables with HRQoL were age, work, bleedings, hospitalization, chronic diseases, hepatitis $\mathrm{C}$ and arthropathy.

Finally, we ran hierarchical regression analyses for all HRQoL scores with socio-demographic and clinical variables entered in the first step and BDI-II score entered in the second step. That way we could examine if depressive symptoms explained HRQoL after controlling for the measured socio-demographic and clinical variables. Socio-demographic and clinical variables entered in the first step explained between $28-51 \%$ of the HRQoL variance. Socio-demographic and clinical variables by themselves explained more variance in physical health domains (49\% in RP, $44 \%$ in PF, $37 \%$ in $\mathrm{GH}, 30 \%$ in BP) and in PCS (51\%) than in mental health domains $(38 \%$ in $\mathrm{SF}, 36 \%$ in RE, $31 \%$ in VT, $28 \%$ in $\mathrm{MH}$ ) and in MCS (29\%). Adding depressive symptoms in the second step significantly increased the amount of explained variance for all HRQoL scores except RP, BP and PCS. The increase was the greatest for MH domain $(27 \%)$ and MCS (26\%), followed by VT $(17 \%)$, RE (13\%) and SF (10\%), in other words in mental health domains and in MCS.

Significant individual predictors for PF were depressive symptoms $(\beta=-0.20, p=0.032)$, for RP chronic conditions $(\beta=-0.26, p=0.005)$, for BP bleedings $(\beta=-0.29$, $\mathrm{p}=0.002)$ and chronic conditions $(\beta=-0.22, \mathrm{p}=0.038)$, for GH income $(\beta=0.23, p=0.045)$, chronic conditions $(\beta=-0.27, p=0.006)$ and depressive symptoms $(\beta=-0.25$, $\mathrm{p}=0.011)$, for VT hospitalization $(\beta=-0.22, \mathrm{p}=0.010)$, chronic conditions $(\beta=-0.24, p=0.009)$, arthropathy $(\beta=0.18, p=0.042)$ and depressive symptoms $(\beta=-0.50$, $\mathrm{p}<0.001)$, for SF household $(\beta=0.27, \mathrm{p}=0.002)$, chronic conditions $(\beta=-0.26, p=0.005)$, arthritis $(\beta=0.16, p=0.045)$ and depressive symptoms $(\beta=-0.39, p<0.001)$, for $\mathrm{RE}$ hospitalization $(\beta=-0.19, \quad \mathrm{p}=0.018)$ and depressive symptoms $(\beta=-0.44, p<0.001)$, for $\mathrm{MH}$ hospitalization $(\beta=-0.23, p=0.004)$ and depressive symptoms $(\beta=-0.62$, $\mathrm{p}<0.001)$, for PCS income $(\beta=0.21, \mathrm{p}=0.039)$, bleedings $(\beta=-0.22, p=0.006)$ and chronic conditions $(\beta=-0.26$, $\mathrm{p}=0.005)$, and for MCS household $(\beta=0.20, p=0.014)$, hospitalization $(\beta=-0.25, \quad p=0.002)$ and depressive symptoms $(\beta=-0.63, \mathrm{p}<0.001)$.

\section{DISCUSSION}

The aim of this study was to examine the association between depressive symptoms and HRQoL in PWH from Croatia and Slovenia. Although the association between depressive symptoms and HRQoL has been examined in several previous studies, as far as we know no study on PWH used BDI-II as a measure of depressive symptoms nor SF-36v2, a newer version of the most common generic HRQoL instrument.

Table 2. Descriptive statistics for HRQoL and correlations with depressive symptoms, socio-demographic and clinical variables

\begin{tabular}{lccrrrrrrrr}
\hline & $\mathrm{PF}$ & $\mathrm{RP}$ & $\mathrm{BP}$ & $\mathrm{GH}$ & $\mathrm{VT}$ & $\mathrm{SF}$ & $\mathrm{RE}$ & $\mathrm{MH}$ & $\mathrm{PCS}$ & $\mathrm{MCS}$ \\
\hline $\mathrm{M}$ & 44.70 & 44.08 & 45.22 & 47.41 & 52.31 & 47.58 & 47.36 & 50.82 & 43.92 & 51.60 \\
SD & 10.62 & 10.72 & 10.75 & 11.41 & 8.54 & 10.20 & 10.64 & 8.69 & 10.10 & 9.41 \\
BDI-II & $-0.46^{*}$ & $-0.44^{*}$ & $-0.42^{*}$ & $-0.54^{*}$ & $-0.63^{*}$ & $-0.57^{*}$ & $-0.61^{*}$ & $-0.70^{*}$ & $-0.42^{*}$ & $-0.68^{*}$ \\
Age & $-0.49^{*}$ & $-0.34^{*}$ & $-0.31^{*}$ & $-0.35^{*}$ & -0.18 & $-0.34^{*}$ & $-0.19^{*}$ & -0.12 & $-0.49^{*}$ & -0.07 \\
Education & $0.26^{*}$ & $0.36^{*}$ & $0.21^{*}$ & $0.35^{*}$ & 0.17 & $0.27^{*}$ & $0.33^{*}$ & $0.20^{*}$ & $0.32^{*}$ & $0.22^{*}$ \\
Household & $0.28^{*}$ & $0.20^{*}$ & 0.17 & 0.18 & $0.23^{*}$ & $0.26^{*}$ & 0.15 & 0.16 & $0.25^{*}$ & 0.15 \\
Income & $0.49^{*}$ & $0.54^{*}$ & $0.35^{*}$ & $0.53^{*}$ & $0.39^{*}$ & $0.43^{*}$ & $0.53^{*}$ & $0.41^{*}$ & $0.51^{*}$ & $0.40^{*}$ \\
Work & $-0.45^{*}$ & $-0.42^{*}$ & $-0.33^{*}$ & $-0.35^{*}$ & $-0.29^{*}$ & $-0.38^{*}$ & $-0.28^{*}$ & $-0.30^{*}$ & $-0.45^{*}$ & $-0.23^{*}$ \\
Bleedings & $-0.35^{*}$ & $-0.35^{*}$ & $-0.42^{*}$ & $-0.28^{*}$ & $-0.28^{*}$ & $-0.31^{*}$ & $-0.27^{*}$ & $-0.22^{*}$ & $-0.41^{*}$ & $-0.19^{*}$ \\
Hospitalization & $-0.28^{*}$ & $-0.43^{*}$ & $-0.26^{*}$ & $-0.24^{*}$ & $-0.42^{*}$ & $-0.40^{*}$ & $-0.49^{*}$ & $-0.47^{*}$ & $-0.26^{*}$ & $-0.49^{*}$ \\
Chronic & $-0.46^{*}$ & $-0.51^{*}$ & $-0.41^{*}$ & $-0.47^{*}$ & $-0.34^{*}$ & $-0.40^{*}$ & $-0.34^{*}$ & $-0.26^{*}$ & $-0.56^{*}$ & $-0.22^{*}$ \\
Arthritis & -0.03 & 0.11 & 0.10 & 0.07 & 0.13 & $0.19^{*}$ & $0.21^{*}$ & 0.16 & 0.01 & $0.24^{*}$ \\
Hepatitis C & $-0.46^{*}$ & $-0.45^{*}$ & $-0.30^{*}$ & $-0.37^{*}$ & $-0.28^{*}$ & $-0.41^{*}$ & $-0.30^{*}$ & $-0.22^{*}$ & $-0.48^{*}$ & $-0.20^{*}$ \\
Arthropathy & $-0.39^{*}$ & $-0.28^{*}$ & -0.13 & -0.13 & -0.03 & -0.14 & -0.12 & -0.07 & $-0.32^{*}$ & 0.02 \\
\hline
\end{tabular}

$* \mathrm{p}<0.05 ; \mathrm{M}=$ arithmetic mean; $\mathrm{SD}=$ standard deviation; $\mathrm{PF}=$ Physical Functioning; $\mathrm{RP}=$ Role-Physical; $\mathrm{BP}=\mathrm{Bodily}$ Pain; $\mathrm{GH}=$ General Health; $\quad \mathrm{VT}=$ Vitality; $\quad \mathrm{SF}=$ Social Functioning; $\quad \mathrm{RE}=$ Role-Emotional; $\mathrm{MH}=$ Mental Health; PCS = Physical Component Summary; MCS = Mental Component Summary 
Table 3. Regression beta coefficients from the last step of hierarchical regression analyses for HRQLL scales and model summary for all steps

\begin{tabular}{|c|c|c|c|c|c|c|c|c|c|c|}
\hline & $\mathrm{PF}$ & $\mathrm{RP}$ & BP & $\mathrm{GH}$ & VT & SF & $\mathrm{RE}$ & MH & PCS & MCS \\
\hline \multicolumn{11}{|l|}{ Step 1} \\
\hline F1 $(11,90)$ & $8.16^{*}$ & $9.67^{*}$ & $4.93^{*}$ & $6.30 *$ & $5.04 *$ & $6.70 *$ & $6.24 *$ & $4.58 *$ & 10.71 & $4.67 *$ \\
\hline Adjusted $\mathrm{R}^{2}$ & 0.44 & 0.49 & 0.30 & 0.37 & 0.31 & 0.38 & 0.36 & 0.28 & 0.51 & 0.29 \\
\hline Age & -0.21 & 0.01 & 0.05 & -0.12 & 0.13 & -0.01 & 0.04 & 0.15 & -0.14 & 0.16 \\
\hline Education & 0.08 & 0.12 & -0.01 & 0.14 & -0.06 & 0.12 & 0.13 & 0.02 & 0.08 & 0.06 \\
\hline Household & 0.09 & 0.07 & 0.11 & 0.03 & 0.26 & $0.27 *$ & 0.07 & .017 & 0.07 & $0.20 *$ \\
\hline Income & 0.17 & 0.16 & 0.07 & $0.23 *$ & -0.09 & -0.10 & 0.11 & -0.04 & $0.21 *$ & -0.10 \\
\hline Work & -0.03 & -0.12 & -0.22 & -0.02 & -0.17 & -0.13 & -0.02 & -0.19 & -0.10 & -0.12 \\
\hline Bleedings & -0.10 & -0.13 & $-0.29 *$ & -0.06 & -0.02 & -0.04 & -0.01 & 0.05 & $-0.22 *$ & 0.08 \\
\hline Hospitalization & -0.01 & -0.15 & -0.04 & 0.05 & $-0.22 *$ & -0.12 & $-0.19^{*}$ & $-0.23^{*}$ & 0.02 & $-0.25^{*}$ \\
\hline Chronic & -0.13 & $-0.26^{*}$ & $-0.22 *$ & $-0.27 *$ & $-0.24^{*}$ & $-0.26^{*}$ & -0.15 & -0.12 & $-0.26^{*}$ & -0.15 \\
\hline Arthritis & 0.00 & 0.12 & 0.07 & 0.07 & 0.06 & $0.16^{*}$ & 0.14 & 0.07 & 0.05 & 0.13 \\
\hline Hepatitis C & -0.06 & -0.10 & -0.05 & -0.01 & 0.01 & -0.05 & 0.03 & 0.12 & -0.11 & 0.09 \\
\hline Arthropathy & -0.17 & -0.06 & -0.07 & 0.04 & $0.18 *$ & 0.09 & -0.02 & 0.02 & -0.06 & 0.11 \\
\hline \multicolumn{11}{|l|}{ Step 1} \\
\hline F2 $(12,89)$ & $8.19^{*}$ & $9.07^{*}$ & $4.62 *$ & $6.70^{*}$ & $8.63^{*}$ & $8.80^{*}$ & $9.20 *$ & $11.09 *$ & $9.76^{*}$ & $11.45^{*}$ \\
\hline Adjusted $\mathrm{R}^{2}$ & 0.46 & 0.49 & 0.30 & 0.40 & 0.48 & 0.48 & 0.49 & 0.55 & 0.51 & 0.55 \\
\hline BDI-II & $-0.20 *$ & -0.12 & -0.11 & $-0.25^{*}$ & $-0.50 *$ & $-0.39 *$ & $-0.44 *$ & $-0.62 *$ & -0.04 & $-0.63 *$ \\
\hline
\end{tabular}

$* \mathrm{p}<0.05 ; \quad \mathrm{F}=$ F-ratio; $\quad \mathrm{PF}=$ Physical Functioning; $\mathrm{RP}=$ Role-Physical; $\quad \mathrm{BP}=$ Bodily Pain; $\mathrm{GH}=$ General Health; VT $=$ Vitality; $\quad \mathrm{SF}=$ Social Functioning; $\quad \mathrm{RE}=$ Role-Emotional; $\quad \mathrm{MH}=$ Mental Health; $\quad \mathrm{PCS}=$ Physical Component Summary; MCS $=$ Mental Component Summary

As hypothesized, depressive symptoms were significantly negatively associated with all HRQoL domains and both component scores. This is also in line with Kim et al. study findings of significant negative associations between BDI and all four domains of the QoL (Kim et al. 2013). As for HRQoL scores, almost all HRQoL norm-based scores of our participants were below 50 indicating poor HRQoL in PWH. Our finding is in line with other comparisons of HRQoL with general populations, for example Naous et al. study using SF-36, as we did, found that PWH in Lebanon had worse score in all domains expect VT (Naous et al. 2019). Our participants had better scores in the mental health than in the physical health domains, and in MCS score compared to PCS score which is also in line with previous findings on $\mathrm{PWH}$ (Kempton et al. 2018b, Krishnan et al. 2015, CuestaBarriuso et al. 2018, Windyga et al. 2014).

Hierarchical regression analyses for all HRQoL scores showed that socio-demographic and clinical variables entered in the first step explained significant percentage of HRQoL variance, ranging between 28$49 \%$ for domain scores. Socio-demographic and clinical variables explained $51 \%$ of PCS, but only $29 \%$ of MCS. In the second step depressive symptoms were entered and explained additional variance for all HRQoL scores except for RP and BP domains, and PCS. Smallest contribution of depressive symptoms for explaining HRQoL variance was observed for PF and $\mathrm{GH}$ domain scores (2-3\%), while that contribution was much larger for mental health domain scores (ranging between 10-27\%) and MCS (26\%). There are only few studies that we can compare these findings with. Kim et al. found that BDI scores were significantly and negatively associated with all four domains of the QoL in regression analyses (Kim et al. 2013). Their measure of QoL was shorter (24 items) and had less differentiation of QoL domains. Rambod et al. found, using haemophilia-specific quality of life measure (Haem-A-QoL) and Depression Anxiety Stress Scales (DASS), that severity of pain and depression were significant predictors of HRQoL and explained $47 \%$ of the variance (Rambod et al. 2018). In our study, which has information about different aspects of HRQoL, amount of explained variance differed depending on the domain being predicted and significant predictors differed as well. Depressive symptoms were significant predictors for six domain scores (PF, GH, VT, SF, RE, MH) and one component score (MCS), followed by having chronic conditions, which was a significant predictor for four domain scores (RP, BP, GH, VT) and one component score (PCS), and hospitalization, which predicted three domain scores (VT, RE, MH) and one component score (MCS).

This study adds to the body of literature examining the association between depressive symptoms and HRQoL in PWH from two novel populations. By using BDI-II, which is an instrument designed and validated for diagnosing and screening of depression, we obtained more valid examination of the prevalence of depressive symptoms among PWH and their association with HRQoL. By using SF-36v2, which measures eight specific HRQoL domains and two component scores, we obtained more detailed information about the 
HRQoL of PWH and could examine predictors of each domain. Our findings indicate that chronic conditions are the most important predictor of physical aspects of HRQoL, with PWH who have more chronic conditions reporting worse physical HRQoL. Since most of the participants were on prophylaxis, only $16 \%$ were hospitalized in the last six months, but hospitalization was an important predictor of mental aspects of HRQoL. Finally, having depressive symptoms was associated with HRQoL of PWH, more so in mental health than in physical health domains. This is even more important in the light that nowadays low priority is given to mental health (Jakovljević 2016). In addition, two recent reviews have shown how important it is to control for depression among patients with chronic diseases. Read et al. have found that depression is two to three times more likely in people with multimorbidity (Read et al. 2017). DeJean et al. found that depression is a common complication of a chronic disease which can worsen the disease, while treating it may lead to better management of the disease and better quality of life (DeJean et al. 2013).

Our findings can be helpful for healthcare professionals working with PWH since they indicate with which specific HRQoL domains depressive symptoms are associated with in PWH. Although it is expected that PWH with more depressive symptoms have lower HRQoL in mental health domains, it is interesting that we found that depressive symptoms are associated with lower HRQoL in some physical health domains as well. In general, with our set of predictors more variance was explained in the mental health than in the physical health domains. Adherence to treatment, chronic pain level and financial issues have all been shown to predict variance in HRQoL in PWH (Bago et al. 2021, Carvalhosa et al. 2014, McLaughlin et al. 2017).

There are of course several limitations of this study. Cross-sectional design of the study does not enable us to draw any conclusion about the direction of influence between depressive symptoms and HRQoL. We combined samples from two populations, which enabled us to test our hypotheses, but we acknowledge that there are possibly differences between them because they come from somewhat different health systems. Results on SF-36v2 were compared to US general population norms because there are no SF36v2 norms available for Croatia and Slovenia, and that also could have an impact on our results. Some studies have shown that MH and MCS scores can be used to screen for depression (Matcham et al. 2016, Walsh et al. 2006), so it can be argued that the associations found between depressive symptoms and mental health domains are due to the fact that BDI-II and MCS measure the same underlying construct. However, in this study we also found that depressive symptoms are associated with HRQoL scales which are not part of MCS score (PF and GH), which do not have this validity problem.

\section{CONCLUSIONS}

This is the first study that examined the association between depressive symptoms and HRQoL in PWH using BDI-II and SF-36v2. As hypothesized, depressive symptoms were significantly negatively associated with all HRQoL domains and both component scores. Participants in this study had better scores in the mental health domains than in the physical health domains with almost all HRQoL norm-based scores below 50 indicating poor HRQoL in PWH. Hierarchical regression analyses for all HRQoL scores showed that socio-demographic and clinical variables explained $28-49 \%$ of HRQoL variance for domain scores, $51 \%$ for PCS, but only $29 \%$ for MCS. Depressive symptoms explained additional variance for all HRQoL scores except for RP and BP domains and PCS, with incremental variance being larger for mental health domain scores (ranging between 10-27\%) and MCS (26\%). This study's findings support that having depressive symptoms is associated with HRQoL of PWH, more in mental health than in physical health domains. Therefore, it would be beneficial for both patients and the public health system if screening for depressive symptoms would be included as a part of the treatment protocol.

\section{Acknowledgements:}

The authors would like to thank all the participants who took part in the study. The authors would also like to thank Bianca Dobson for language editing of this publication.

\section{Conflict of interest: None to declare.}

\section{Contribution of individual authors:}

Martina Bago: study design, data collection, writing the manuscript.

Ana Butković: statistial analysis, literature search, writing the manuscript.

Barbara Faganel Kotnik: data collection, writing the manuscript.

Ivana Prga \& Vesna Bačić Vrca: literature search, writing the manuscript.

Silva Zupančić Šalek: data collection, writing the manuscript.

\section{References}

1. Bago M, Butkovic A, Preloznik Zupan P, Faganel Kotnik B, Prga I, Vrca VB, Salek SZ: Association between reported medication adherence and health-related quality of life in adult patients with haemophilia. Int J Clin 2021; 29:1-8

2. Bago M, Butkovic A, Preloznik Zupan I, Faganel Kotnik B, Prga I, Bacic Vrca V et al.: Depressive symptoms and adherence to prophylaxis in patients with haemophilia from Croatia and Slovenia. Haemophilia 2020; 26:e161-7 
3. Barlow JH, Stapley J, Ellard DR \& Gilchrist M: Information and self-management needs of people living with bleeding disorders: a survey. Haemophilia 2007; 13:264-70

4. Batt K, Boggio L, Neff A, Buckner TW, Wang M, Quon D et al.: Patient-reported outcomes and joint status across subgroups of US adults with hemophilia with varying characteristics: Results from the Pain, Functional Impairment, and Quality of Life (P-FiQ) study. Eur J Haematol 2018; 100:14-24

5. Beck A, Steer R \& Brown G: Manual for Beck Depression Inventory-II. TX: Psycholoical Corporation, San Antonio, 1996

6. Buckner TW, Batt K, Quon D, Witkop M, Recht M, Kessler $C$ et al.: Assessments of pain, functional impairment, anxiety, and depression in US adults with hemophilia across patient-reported outcome instruments in the Pain, Functional Impairment, and Quality of Life (P-FiQ) study. Eur J Haematol 2018; 100: 5-13

7. Canclini M, Saviolo-Negrin N, Zanon E, Bertoletti $R$, Girolami A \& Pagnan A: Psychological aspects and coping in haemophilic patients: a case-control study. Haemophilia 2003; 9: 619-24

8. Carvalhosa AM, Henrard S, Lambert $C$ \& Hermans $C$. Physical and mental quality of life in adult patients with haemophilia in Belgium: the impact of financial issues. Haemophilia. 2014; 20: 479-85

9. Castaño AF, Restrepo MJ \& Durán FS: Quality of life in a population with haemophilia: A cross-sectional study from a single haemophilia treatment center. Rev Colomb Reumatol 2017; 24:18-24

10. Cavazza M, Kodra Y, Armeni P, De Santis M, LopezBastida J, Linertova $R$ et al.: Social/economic costs and quality of life in patients with haemophilia in Europe. Eur J Health Econ 2016; 17:53-65

11. Centers for Disease Control and Prevention: Measuring healthy days. CDC: Atlanta, Georgia, 2000

12. Cuesta-Barriuso $R$, López-Pina JA, Nieto-Munuera J, Sagarra-Valls G, Panisello-Royo JM \& Torres-Ortuño A: Effectiveness of the Medtep Hemophilia online platform for adherence to prophylactic treatment in haemophilia patients: Results from a 1-year observational study. Haemophilia 2018; 24:452-9

13. DeJean D, Giacomini M, Vanstone $M$, \& Brundisini F: (2013). Patient experiences of depression and anxiety with chronic disease: a systematic review and qualitative meta-synthesis. Ontario Health Technol Assess Ser $2013 ; 13: 1$

14. Gerontoukou E-I, Michaelidoy S, Rekleiti M, Saridi M \& Souliotis $K$ : Investigation of anxiety and depression in patients with chronic diseases. Health Psychol Res 2015; $3: 2123$

15. Hermans $C$, Klamroth $R$, Richards $M$, de Moerloose $P$, Garrido RP \& the EHTSB: Outcome measures in European patients with haemophilia: survey of implementation in routine clinical practice, perception of relevance and recommendations by European treaters in the EHTSB. Haemophilia 2017; 23: 222-9

16. Iannone M, Pennick L, Tom A, Cui H, Gilbert M, Weihs K et al.: Prevalence of depression in adults with haemophilia. Haemophilia 2012; 18:868-74

17. IBM Corp: IBM SPSS Statistics for Windows, Version 26.0. Armonk, NY: IBM Corp., Released 2019
18. Jakovljević M: Public and global mental health promotion for empathic civilisation: A new goal of Psychiatria Danubina. Psychiatr Danub 2016; 28:312-4

19. Kempton CL, Buckner TW, Fridman M, Iyer NN \& Cooper DL: Factors associated with pain severity, pain interference, and perception of functional abilities independent of joint status in US adults with hemophilia: multivariable analysis of the Pain, Functional Impairment, and Quality of Life (P-FiQ) study. Eur J Haematol 2018a; 100:25-33

20. Kempton CL, Recht M, Neff A, Wang M, Buckner TW, Soni A et al.: Impact of pain and functional impairment in US adults with haemophilia: patient-reported outcomes and musculoskeletal evaluation in the Pain, Functional Impairment and Quality of Life (P-FiQ) study. Haemophilia 2018b; 24: 261-70

21. Kim S-Y, Kim S-W, Kim J-M, Shin I-S, Baek H-J, Lee H-S et al.: Impact of personality and depression on quality of life in patients with severe haemophilia in Korea. Haemophilia 2013; 19: e270-5

22. Krishnan S, Vietri J, Furlan R \& Duncan N: Adherence to prophylaxis is associated with better outcomes in moderate and severe haemophilia: results of a patient survey. Haemophilia 2015; 21:64-70

23. Limperg PF, Terwee CB, Young NL, Price VE, Gouw SC, Peters $M$ et al.: Health-related quality of life questionnaires in individuals with haemophilia: a systematic review of their measurement properties. Haemophilia 2017; 23:497-510

24. Mandić JJ, Ivkić PK, Mandić K, Lešin D, Jukić $T$ \& Jurčević JP: Quality of life and depression level in patients with watery eye. Psychiatr Danub 2018; 30:471-7

25. Maruish ME (Ed.): User's manual for the SF-36v2 Health Survey (3rd ed.). Lincoln, RI: QualityMetric Incorporated, 2011

26. Matcham F, Norton S, Steer S \& Hotopf M: Usefulness of the SF-36 Health Survey in screening for depressive and anxiety disorders in rheumatoid arthritis. BMC Musculoskelet Disord 2016; 17:224

27. McLaughlin JM, Munn JE, Anderson TL, Lambing A, Tortella $B$ \& Witkop ML. Predictors of quality of life among adolescents and young adults with a bleeding disorder. Health Qual Life Outcomes 2017; 15:1-9

28. Miners AH, Sabin CA, Tolley KH, Jenkinson C, Kind P \& Lee CA: Assessing health-related quality-of-life in individuals with haemophilia. Haemophilia 1999; 5:378-85

29. Moussavi S, Chatterji S, Verdes E, Tandon A, Patel V \& Ustun B: Depression, chronic diseases, and decrements in health: results from the World Health Surveys. Lancet 2007; 370: 851-8

30. Naous E, de Moerloose P, Sleilaty G, Casini A \& Djambas Khayat $C$ : The impact of haemophilia on the social status and the health $\square$ related quality of life in adult Lebanese persons with haemophilia. Haemophilia 2019; 25: 264-9

31. PASS 15 Power Analysis and Sample Size Software NCSS, LLC. Kaysville, Utah, USA; https//ncss.com/software/pass 2017

32. Pinto PR, Paredes AC, Moreira P, Fernandes S, Lopes M, Carvalho $M$ et al.: Emotional distress in haemophilia: factors associated with the presence of anxiety and depression symptoms among adults. Haemophilia 2018a; 24: e344-e353

33. Pinto P, Paredes A, Pedras S, Costa P, Crato $M$, Fernandes $S$ et al.: Sociodemographic, clinical, and 
psychosocial characteristics of people with hemophilia in Portugal: findings from the First National Survey. TH Open 2018b; 2:e54-e67

34. Poon JL, Zhou Z-Y, Doctor JN, Wu J, Ullman MM, Ross C et al.: Quality of life in haemophilia A: Hemophilia Utilization Group Study Va (HUGS-Va). Haemophilia 2012; 18:699-707

35. Rambod $M$, Sharif $F$, Molazem Z, Khair $K$ \& von Mackensen S: Health-related quality of life and psychological aspects of adults with hemophilia in Iran. Clin Appl Thromb 2018; 24:1073-81

36. Read JR, Sharpe L, Modini M \& Dear BF: Multimorbidity and depression: a systematic review and meta-analysis. $J$ Affect Disord 2017; 221:36-46

37. Ryu E: Effects of skewness and kurtosis on normal-theory based maximum likelihood test statistic in multilevel structural equation modeling. Behav Res Methods 2011; 43:1066-74

38. Tran DQ, Barry $V$, Antun A, Ribeiro $M$, Stein $S \&$ Kempton CL: Physician trust and depression influence adherence to factor replacement: a single-centre crosssectional study. Haemophilia 2017; 23: 98-104.

39. Triemstra AHM, Van der Ploeg HM, Smit C, Briët E, Adèr $H J \&$ Rosendaal FR: Well-being of haemophilia patients: a model for direct and indirect effects of medical parameters on the physical and psychosocial functioning. Soc Sci Med 1998; 47:581-93

40. Varaklioti A, Kontodimopoulos $N$, Katsarou $O \&$ Niakas D: Psychometric properties of the Greek Haem-A-QoL for measuring quality of life in Greek haemophilia patients. BioMed Res Int 2014; 968081:1-12

41. Walsh TL, Homa K, Hanscom B, Lurie J, Sepulveda MG $\& A b d u, W:$ (2006). Screening for depressive symptoms in patients with chronic spinal pain using the SF-36 Health Survey. Spine J 2006; 6:316-20

42. Windyga J, Lin VW, Epstein JD, Ito D, Xiong Y, Abbuehl $B E$ et al.: Improvement in health-related quality of life with recombinant factor $I X$ prophylaxis in severe or moderately severe haemophilia $B$ patients: results from the BAX326 Pivotal Study. Haemophilia 2014; 20:362-8

Correspondence:

Martina Bago, MPharm

Andrija Stampar Teaching Institute of Public Health

Mirogojska cesta 16, 10000 Zagreb, Croatia

E-mail:martina.bago.hr@gmail.com 